\title{
Experimental Modal Analysis of Violins Made from Composites ${ }^{\dagger}$
}

\author{
Tim Duerinck ${ }^{1,2,3, *}$, Mathias Kersemans ${ }^{1}$, Ewa Skrodzka ${ }^{4}$, Marc Leman ${ }^{2}$, Geerten Verberkmoes ${ }^{3}$ \\ and Wim Van Paepegem ${ }^{1}$ \\ 1 Department of Materials, Textiles and Chemical Engineering (MaTCh), Ghent University, 9000 Gent, \\ Belgium; mathias.kersemans@ugent.be (M.K.); wim.vanpaepegem@ugent.be (W.V.P.) \\ 2 Department of Art, Music and Theatre Sciences, Ghent University, 9000 Gent, Belgium; \\ marc.leman@ugent.be \\ 3 Department of Design, Hogent, 9000 Gent, Belgium; geerten.verberkmoes@hogent.be \\ 4 Faculty of String Instruments, Harp, Guitar and Violin-Making, I.J. Paderewski Academy of Music, \\ 61-808 Poznań, Poland; afa@amu.edu.pl \\ * Correspondence: tim.duerinck@ugent.be; Tel.: +32-492-56-60-49 \\ + Presented at 18th International Conference on Experimental Mechanics (ICEM18), Brussels, Belgium, \\ 1-5 July 2018.
}

Published: 19 July 2018

\begin{abstract}
Six prototype violins made from composite materials are made and investigated using experimental modal analysis with the roving hammer method. The average FRF's obtained show an influence of the materials on the vibrational response up to $2200 \mathrm{~Hz}$. The A0 breathing mode and B1- mode are identified and are found to be significantly lower than in classical wooden violins. Additional measurements with a Laser Doppler Vibrometer and shaker found the same modes with a small difference in frequency $(3-8 \mathrm{~Hz})$.
\end{abstract}

Keywords: violin; modal analysis; composite

\section{Introduction}

The vibrational modes of wooden music instruments like the guitar and violin are well documented in literature, and provide insight in the dynamics of the instrument [1,2]. Although there is discussion about the link between the vibrational modes and the actual sound radiation [3], an experimental modal analysis can explain differences in the radiated sound parameters. For example, a different placement of the bassbar and lack of arching in a trapezoidal violin can result in more symmetric vibrational modes (Figure 1), which on their turn result in a lack of brilliance in the sound [4]. As such, experimental modal analysis is a valuable tool to examine the performance of experimental music instruments.

Recently, instruments made from fiber reinforced polymers (or composites) have successfully entered the market. Companies such as mezzo-forte Streichinstrumente, Luis and Clark and Elixir Violins are producing carbon fibre reinforced polymer (CFRP) violins, whilst BlackbirdGuitar makes guitars and ukuleles from both carbon and flax fiber reinforced polymers. Composite materials seem a compatible alternative to tonewood in sound production and especially durability, yet the research on composite music instruments is a very new field and mostly limited to the making and comparing of one prototype to a classic wooden instrument [5]. However, none of these publications compare instruments made from different composites and asses which changes in vibrational behavior are due to the effect of geometrical design and which are the effect of the used material. 

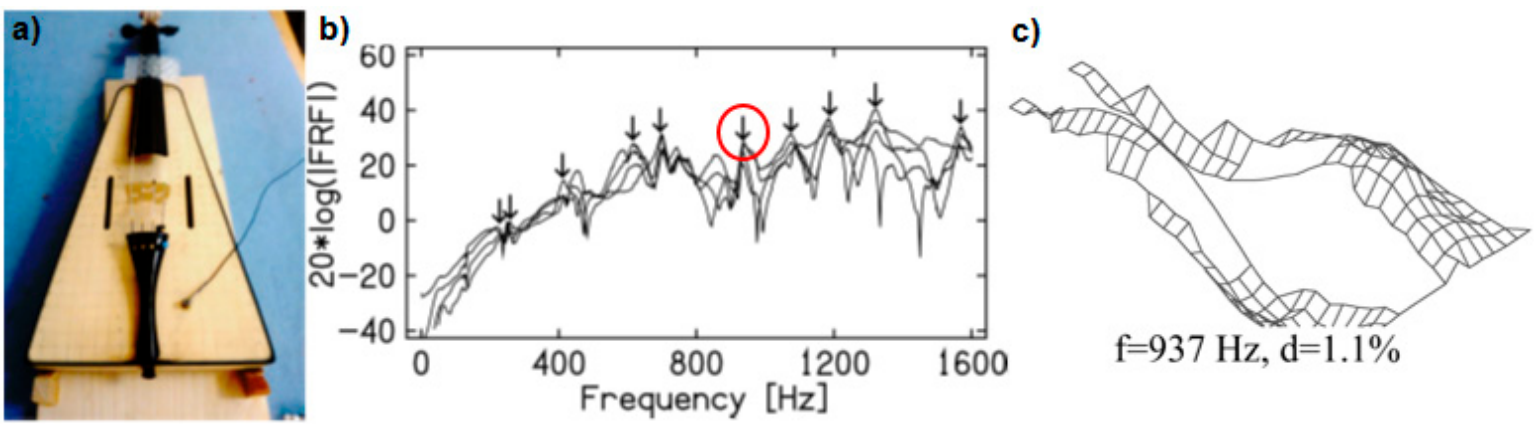

Figure 1. (a) Trapezoidal violin with accelerometer, (b) FRF function of 4 points on the trapezoidal violin, (c) the vibration mode on $937 \mathrm{~Hz}$.

\section{The Prototype Violins}

To objectively assess the influences of different composites on the vibrational behavior and sound of violins, six prototype violins where constructed in the laboratory. The model is based on that of a classic violin (Stradivari 1716), but adapted to make mold fabrication possible. The violins have the same geometry (apart from inevitable manufacturing variability). The ribs, back plate and neck of all the violins is made from woven carbon composite. The soundboards (or top plates) are made from different materials. All composite parts were made using vacuum assisted resin transfer method (VARTM). Figure 2 shows the moulds used for construction of the prototypes (a) and the top plate under vacuum before infusion.



Figure 2. (a) Mould for body (left) and top plate (right), (b) top plate production through VARTM.

The thickness of the soundboard of a classic violin is made so it is rigid enough to withstand the pressure of the strings without collapsing over time, as well as being flexible enough to vibrate easily [6]. For this purpose the required thickness of the soundboards for each material was calculated using laminate theory. In this way all top plates have a similar bending stiffness as classic violin along the axis.

Figure 3 shows the six manufactured violins: 1 . Woven carbon composite (hereafter named CTA), 2. Unidirectional carbon composite (CUD), 3. Classical tonewood (SPRUCE), 4. Unidirectional flax composite (FLAX), 5. Carbon-Nomex Sandwich (HC) and 6. again woven carbon composite (CTB). Instrument nr. 6 was included to assess the reproducibility of manufacturing and experiment results. In this way, we can study the effects of the material type on the vibrational performance of the soundboards. 


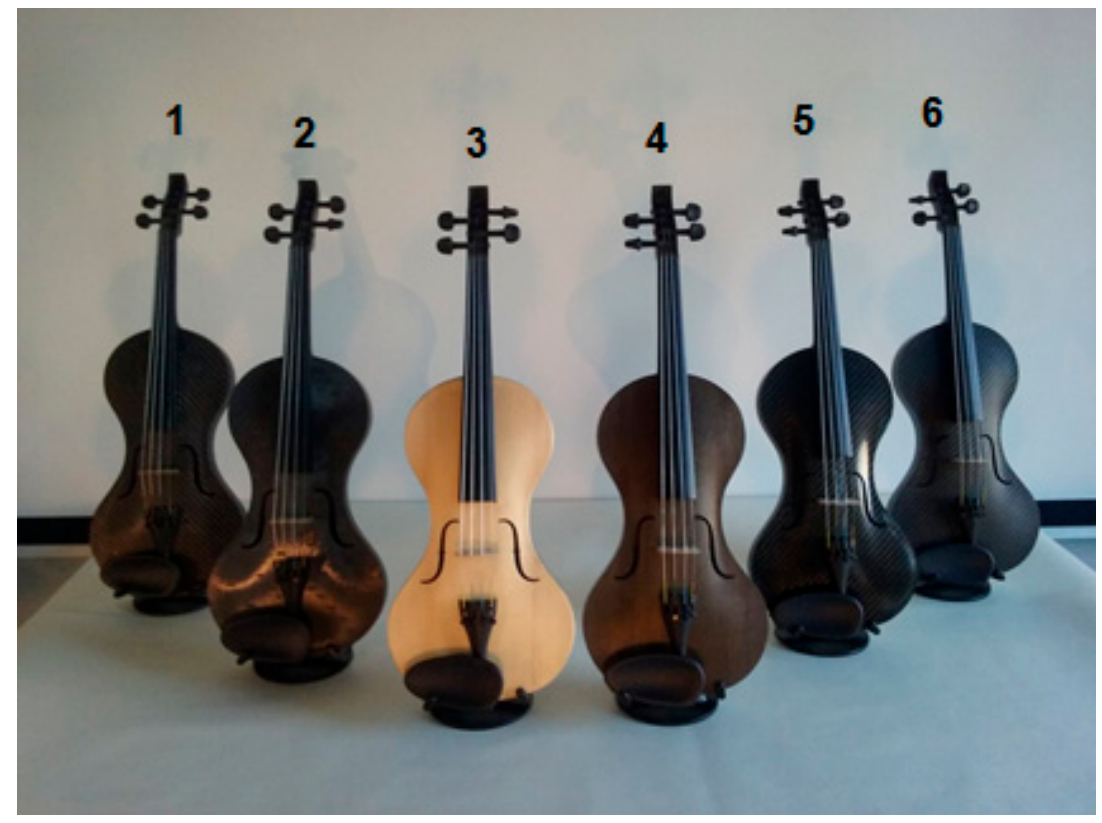

Figure 3. Six prototype violins with soundboards made from different materials.

\section{Modal Experiment}

Experimental modal analysis describes the dynamics of any vibrating system in terms of modal parameters. For analyses of music instruments, the natural frequencies and damping, as well as deformation patterns (mode shapes) associated with them are thought to have a large effect on the sound produced [3,7]. Experimental modal analysis can be performed in many ways. For our first analysis of the composite violins we chose the method of a roving impact hammer and a fixed response measurement point, as this method has been found to work well for music instruments and is already well described in previous papers [8-10]. On each violin, 348 predetermined points were marked as shown in Figure 4a, the distance between the points on the grid is $10 \mathrm{~mm}$.

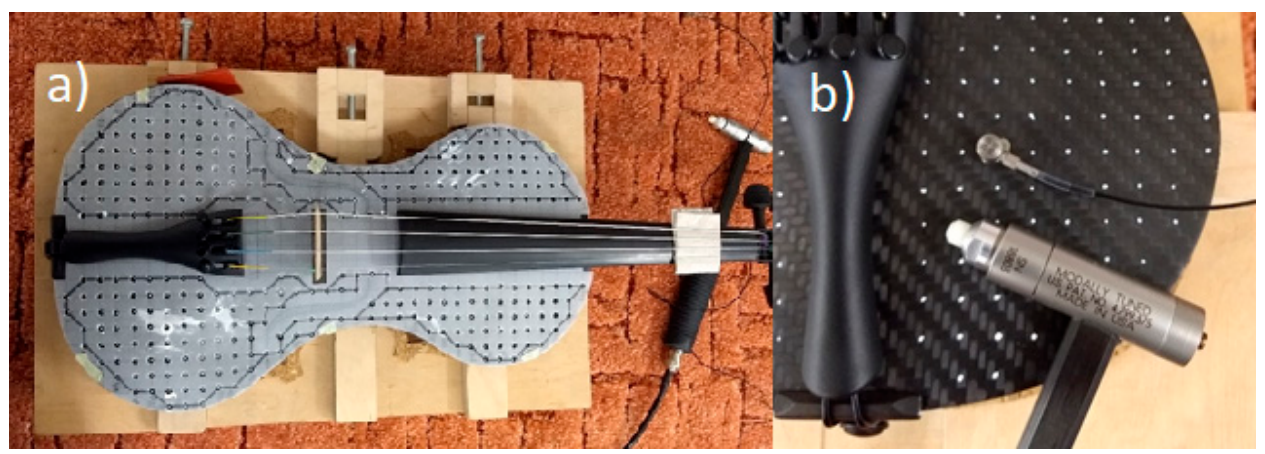

Figure 4. (a) Grid on top of one of the violins for marking the points, (b) mounted accelerometer on point 84 and impact hammer.

The violins where fixed in a mold which clamped the instrument on its edges, as shown in Figure 4a. An ONOSOKKI NP-2910 accelerometer, with a mass of $2 \mathrm{~g}$ and sensitivity of $0.3 \mathrm{pC} / \mathrm{m} / \mathrm{s} 2$ was mounted with bee-wax on point $\mathrm{nr}$. 84. The instrument was excited on all points by an impact hammer (PCB Impact Hammer 086C05; sensitivity $2.25 \mathrm{mV} / \mathrm{N}$ ). With 4096 FFT lines and a measurement range of $0-3200 \mathrm{~Hz}$ a spectral resolution of $2 \mathrm{~Hz}$ was reached. The quality of the measurement was controlled by means of the coherence and five averages. If the coherence was not consistently close to 1 the measurement was repeated. From all FRF's measured, the SMS STARModal R software package calculated possible modes. Based on the stability of the frequency and damping, as well as the estimated damping, stable modes where selected for further examination. 
From each violin, the same 10 measurements points where used to calculate average FRF's from all violins, as displayed in Figure 5. To make it easier to differentiate amplitude differences between the different violins, averages where taken in $200 \mathrm{~Hz}$ bands.

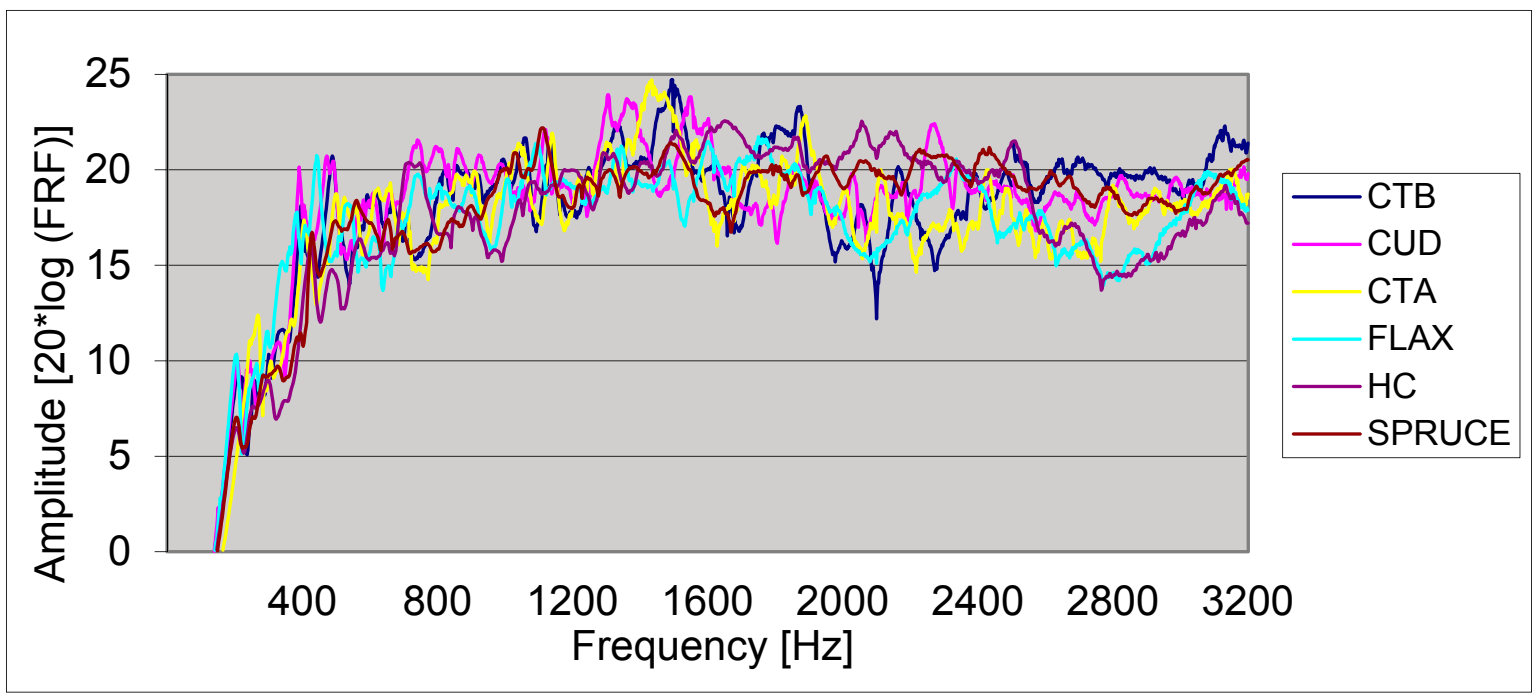

Figure 5. Average FRF of all prototype violins.

\section{Results and Discussion}

As we can see in Figure 6, the average amplitudes in $200 \mathrm{~Hz}$ bands of CTA and CTB, which are made to be identical, show only small differences up to $2200 \mathrm{~Hz}$. The top plates made from other materials, show larger amplitude differences. These results seem to indicate that material properties have a distinct and repeatable influence on the amplitude of violins up to $2200 \mathrm{~Hz}$.

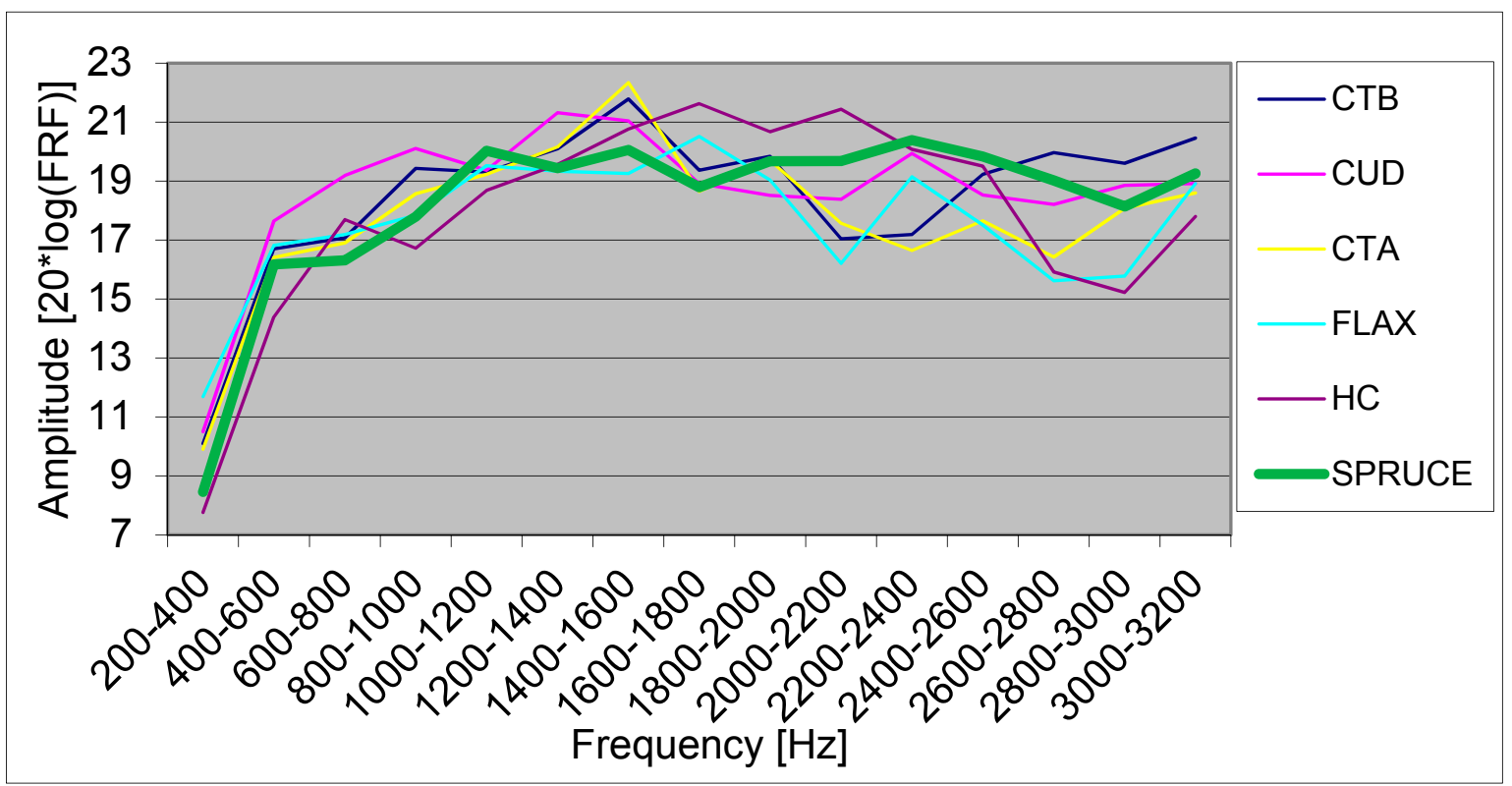

Figure 6. Average FRF of all prototype violins in $200 \mathrm{~Hz}$ bands.

Spruce, the material used normally for soundboards, has a rather low amplitude up to $1000 \mathrm{~Hz}$, after which the average amplitude remains quite stable. HC starts off with the lowest amplitude up to $1400 \mathrm{~Hz}$, with the only exception between $600-800 \mathrm{~Hz}$. The material then shows a peak between 1600 and $2200 \mathrm{~Hz}$, after which the amplitude drops again rapidly. Flax starts off with the highest average amplitude in 200-400 Hz. It then shows only small differences with spruce up to $2000 \mathrm{~Hz}$. CTA and CTB both show a peak between $1400-1600 \mathrm{~Hz}$, after which they both drop until they 
separate around $2200 \mathrm{~Hz}$. CUD shows a higher amplitude in the lower frequencies, especially between $600-1000 \mathrm{~Hz}$, after which the differences with spruce become less distinct.

The mode shapes of a violin in the lower frequencies are well described in literature [3] and are by some believed to have a large influence on the produced sound of the instrument $[7,10]$. Two clear modes could be recognized, the A0 breathing mode and a B1 mode, most likely B1- (see the results in Table 1). A0 is marked by a movement of the entire plate around the bass bar. B1- has a strong motion in the lower bouts, separating it from the upper bouts by a nodal line. The frequency on which these modes are found is lower than usual for violins. A0 is normally found at $275 \pm 9 \mathrm{~Hz}$ and B1- at $476 \pm 16 \mathrm{~Hz}$. As the spruce soundboard shows a similar drop in mode frequencies as the other materials, this is most likely caused by other differences between these prototypes and a classical violin like the design or material of the back plates, ribs or neck.

Table 1. A0 and B1(-) modes found in the prototype violins.

\begin{tabular}{ccccccccccccc}
\hline & CTB & & CUD & & CTA & & Flax & \multicolumn{3}{c}{ HC } & \multicolumn{2}{c}{ Spruce } \\
\hline & Hz & $\%$ & Hz & $\%$ & Hz & $\%$ & Hz & $\%$ & Hz & $\%$ & Hz & $\%$ \\
\hline A0 & 221 & & 200 & & 251 & & 198 & & 192 & & 199 & \\
B1- & 393 & 2.10 & 391 & 2.64 & 409 & 2.43 & 380 & 3.38 & 423 & 2.36 & 424 & 2.57 \\
\hline
\end{tabular}

As the A0 mode is so low, no good estimations could be done on the damping of the specific mode. For B1- however, the only trend that we can see is the higher damping of flax. As CTA and CTB show a difference of $0.54 \%$, this shows that other differences we might observe in the damping of B1- will most likely be caused by other properties than the material. Some research suggests that a lower B1 mode might contribute to a better sounding instrument in classic wooden violins [10]. If this is the case for violins made from different materials as well must be verified through listening tests, which are ongoing.

\subsection{Other Measurement Methods for Modal Analysis of Violins}

Although the previous method has been often used to investigate music instruments, the influence of the accelerometer mounted on the lightweight plate cannot be excluded. Replacing the measurement device (accelerometer) by a Laser Doppler Vibrometer allows for a contactless measurement point. In this case a 3D Infrared Scanning Laser Doppler Vibrometer (Polytec PSV 500 3D HV Xtra) is used, both the in-plane and the out-of-plane vibrational response can be studied. An additional benefit of a scanning laser is that the excitation place can be fixed, which can allow for both easier and more repeatable measurements.

\subsubsection{Excitation through Speaker}

If we wish to have an entirely contactless measurement, we can excite the instrument with a speaker. Different signals where examined: burst chirp, periodic chirp, burst random, pseudo random, sweep and white noise. Of these signals a sweep allowed for the best coherence in a short measurement time ( $0.8 \mathrm{~s}$ per measurement). Although this should be sufficient to find the frequencies on which the modes occur, the exact placement of the speaker to the instrument has a large influence on the amplitude measured by the LDV, as shown in Figure 7. 


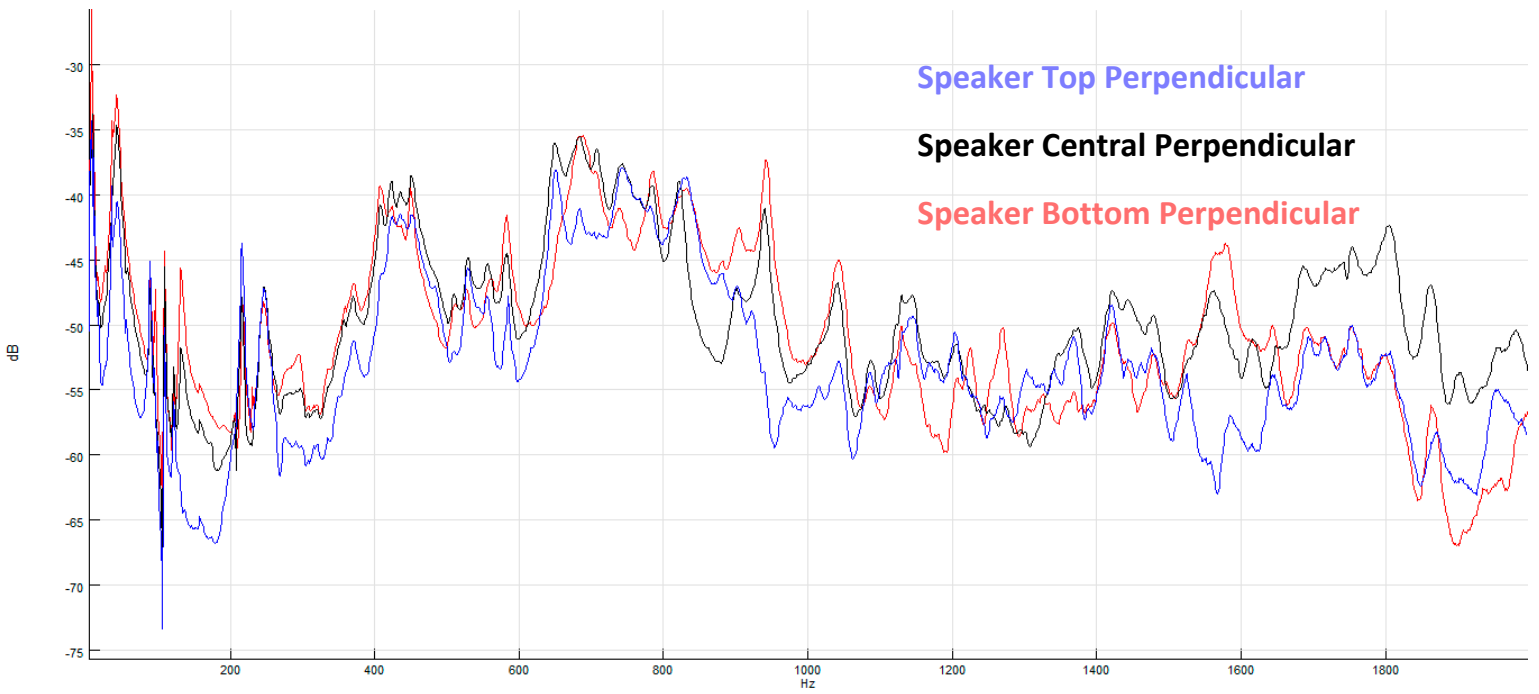

Figure 7. Vibrational response in $\mathrm{dB}$ of different speaker placements for speaker excitation.

\subsubsection{Excitation through Shaker}

By attaching a shaker to a part of the instrument, the measurement is not contactless anymore. However, the shaker can be mounted through a stinger on the bridge of the instrument, which allows for an excitation with a higher ecological validity. Through the shaker, the excitation signal can also be controlled better than through a not-automated modal hammer. CTA was examined again through this method. The A0 breathing mode was now found $8 \mathrm{~Hz}$ lower at $243 \mathrm{~Hz}$ and B1- at $3 \mathrm{~Hz}$ lower at $406 \mathrm{~Hz}(409 \mathrm{~Hz})$. It must be noted however that the gluing of the stinger to the bridge will limit its ability to move freely. Figure 8 displays the measured A0 breathing and the B1- mode shape.
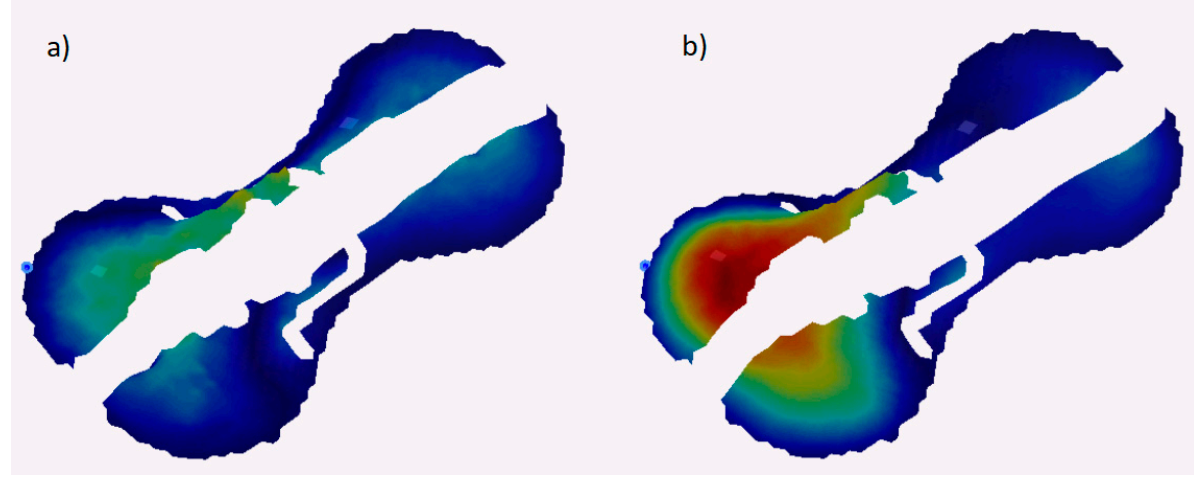

Figure 8. (a) A0 breathing mode at $243 \mathrm{~Hz}$, (b) B1- mode at $406 \mathrm{~Hz}$.

\section{Conclusions}

In our test, material properties seem to have a distinct influence on the amplitude of the vibrations of the instrument up to $2200 \mathrm{~Hz}$. A0 and B1- mode could be identified and were found to be lower than in a conventional wooden violin. Although this must be verified by further testing, large differences in amplitudes between different instruments in specific frequency bands could play a role in the tonal color produced by the instrument.

Author Contributions: Due to the multidisciplinary field of this research, multiple researchers where needed in various stages of the research. T.D., M.K., E.S., M.L., G.V. and W.V.P. cooperated in the design of the experiment and analyses of the data; T.D. made the prototype violins with G.V. as supervisor. T.D. and E.S. set up the experimental modal analysis; T.D. performed the measurements; T.D. wrote the paper.

Acknowledgments: This research is funded by FWO through a fellowship for fundamental research. This includes a fixed bench fee that can be used to publish in open access. 
Conflicts of Interest: The authors declare no conflict of interest. The founding sponsors had no role in the design of the study; in the collection, analyses, or interpretation of data; in the writing of the manuscript, and in the decision to publish the results.

\section{References}

1. Marshall, K.D. Modal analysis of a violin. J. Acoust. Soc. Am. 1985, 77, 695-709, doi:10.1121/1.392338.

2. Torres, J.A.; Boullosa, R.R. Influence of the bridge on the vibration of the top plate of a classic guitar. Appl. Acoust. 2009, 70, 1371-1377, doi:10.1016/j.apacoust.2009.07.002.

3. Bissinger, G. Structural acoustics of good and bad violins. J. Acoust. Soc. Am. 2008, 124, 1764-1773, doi:10.1121/1.2956478.

4. Duerinck, T.; Skrodzka, E.; Linde, B.B.J. Modal analysis of a trapezoidal violin built after the description of F. Savart. Arch. Acoust. 2014, 39, 623-628, doi:10.2478/aoa-2014-0067.

5. Damodaran, A. An Overview of Fibre-Reinforced Composites for Musical Instrument Soundboards. Aust. Acoust. Soc. 2015, 43, 117-122, doi:10.1007/s40857-015-0008-5.

6. Johnson, C.; Courtnall, R.; Menuhin, Y. The Art of Violin Making; Robert Hale: London, UK, 1999.

7. Cough, C. Violin Acoustics. Acoust. Today 2016, 12, 22-30.

8. Skrodzka, E.; Krupa, A.; Rosenfeld, E.; Linde, B.B.J. Mechanical and optical investigation of dynamic behavior of violins in modal frequencies. Appl. Opt. 2009, 48, 165-170, doi:10.1364/AO.48.00C165.

9. Skrodzka, E.; Łapa, A.; Linde, B.B.J.; Rosenfeld, E. Modal parameters of two complete guitars differing in the bracing pattern of the soundboard. J. Acoust. Soc. Am. 2011, 130, 2186-2195, doi:10.1121/1.3626194.

10. Skrodzka, E.; Linde, B.B.J.; Krupa, A. Modal Parameters of Two Violins with Different Varnish Layers and Subjective Evaluation of Their Sound Quality. Arch. Acoust. 2013, 38, 75-81, doi:10.2478/aoa-2013-0009.

(C) 2018 by the authors. Licensee MDPI, Basel, Switzerland. This article is an open access article distributed under the terms and conditions of the Creative Commons Attribution (CC BY) license (http://creativecommons.org/licenses/by/4.0/). 